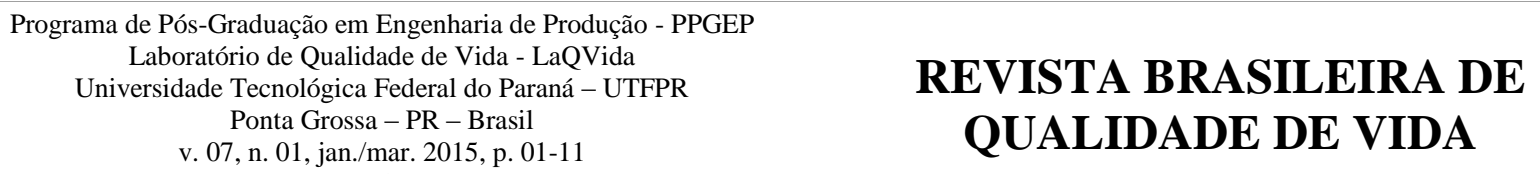

\section{REVISTA BRASILEIRA DE QUALIDADE DE VIDA}

\title{
Avaliação da qualidade de vida relacionada à saúde de participantes do Programa Terceira Idade: Vitalidade e Cidadania
}

\section{Assessing the quality of life related to health of elderly participants in the Elderly Program: Vitality and Citizenship}

\author{
Maria Ruth Gonçalves Gaede-Carrillo \\ Universidade Federal de Ouro Preto - UFOP - Ouro Preto - Minas Gerais - Brasil \\ mrgaede@yahoo.com.br \\ Cristiane de Paula Rezende \\ Universidade Federal de Ouro Preto - UFOP - Ouro Preto - Minas Gerais - Brasil \\ cris7paula@gmail.com \\ Elza Conceição de Oliveira Sebastião \\ Universidade Federal de Ouro Preto - UFOP - Ouro Preto - Minas Gerais - Brasil \\ ecos.71@hotmail.com \\ Nuncio Antônio Araújo Sól \\ Universidade Federal de Ouro Preto - UFOP - Ouro Preto - Minas Gerais - Brasil \\ nunciosol@gmail,com
}

\section{RESUMO}

OBJETIVO: Analisar a percepção dos idosos do Programa Terceira Idade (PTI) em relação a sua qualidade de vida relacionada à saúde (QVRS).

MÉTODOS: Foi realizado um estudo de caráter transversal do tipo inquérito exploratório descritivo aprovado por um Comitê de Ética em Pesquisa. Participaram 66 idosos residentes no município de Ouro Preto praticantes de atividades físicas no Centro Desportivo da Universidade Federal de Ouro Preto. Foram incluídos indivíduos com idade igual ou superior a 60 anos participantes do PTI. Para a coleta de dados utilizou-se um questionário semiestruturado com questões relacionadas às características sociodemográficas e clínicas e o questionário SF-36 para descrever a percepção dos idosos em relação à sua qualidade de vida (QV). Os resultados do SF-36 foram analisados por meio do cálculo da pontuação de cada dimensão para cada participante.

RESULTADOS: Dentre os participantes a maioria eram mulheres $(78,8 \%)$, apresentavam idade entre 60 e 69 anos, referiram ter estudado até 8 anos e eram casados. Quanto às características clínicas, $42,5 \%$ deles relataram usar até dois medicamentos, a comorbidade autorreferida mais prevalente foi a hipertensão $(59,0 \%)$ e $15,2 \%$ dos sujeitos referiram não apresentar nenhuma enfermidade. Os domínios referentes aos aspectos físicos, sociais e emocionais apresentaram pontuação 100,0 para pelo menos $50,0 \%$ dos sujeitos. A pontuação mediana obtida foi acima de 70,0, exceto para o domínio dor, nível considerado como bom para esses participantes específicos.

CONCLUSÕES: Os resultados mostraram que grande parcela dos idosos avaliados era fisicamente ativa e conhecia os benefícios da prática regular de exercícios físicos. Embora os idosos do PTI tenham relatado problemas de saúde crônicos e fizessem uso de polifarmácia, conjectura-se que os escores de QVRS tenham sido resultantes da regularidade das atividades físicas estimuladas pela 
assistência multidisciplinar. Ou seja, idosos participantes de programas de exercícios têm melhor percepção de QV, principalmente quando analisados os componentes relacionados à saúde.

PALAVRAS-CHAVE: Qualidade de vida. Idoso. Saúde do idoso.

\section{ABSTRACT}

OBJECTIVE: Analyze the perception of the Third Age Program (TAP) in relation to their quality of life (HRQOL) related to health.

METHODS: A descriptive exploratory transversal study was performed. This research was approved by an Ethics Committee. Sixty-six people were included as subjects, resident in Ouro Preto/Brazil, over 60y.o. and participants of Federal University of Ouro Preto Centre of Sports. A semi-structured questionnaire was the instrument for data collection of socio-demographic and clinical characteristics. The SF-36 questionnaire was applied to describe the perception of the elderly about their quality of life and calculated its scale scores for each participant.

RESULTS: The main characteristics of the participants were female $(78,8 \%)$, aged 60 to 69 years, over 8 years of education (referred), married people. Clinical characteristics referred drug use of $\leq 2$ items (42,5\%); hypertensive (59,0\%). Of all the subjects, 15,2\% didn't reported any clinical condition. At least $50,0 \%$ of the subjects reached score of 100 in the physical, social and emotional domains. In exception for pain domain, the median score obtained was above 70 , considered as good level for this specific group.

CONCLUSIONS: The results showed that large number of the participants were physically active and knew the benefits of regular physical exercise. Although TAP participants referred chronic health problems and were under polypharmacy, it is conjectured that the HRQOL scores were result of regular physical activities stimulated by the multidisciplinary care. Active elderly have better perception of quality of life, especially when analyzed the components related to health.

KEYWORDS: Quality of life. Aged. Health of the elderly.

\section{Introdução}

A população idosa vem aumentando significativamente sem que haja, na mesma velocidade, o suporte para esse contingente populacional. O envelhecimento é uma questão abordada por pesquisadores nacionais e internacionais, por meio de estudos científicos, revelando a preocupação destes com a maior proporção de idosos no mundo, tendo em conta a expectativa de vida cada vez mais alta (VECCHIA et al., 2005).

O que antes era privilégio de poucos, hoje é um objetivo a ser alcançado pela população mundial, inclusive nos países mais pobres. Os indivíduos estão conseguindo viver mais, para que estes anos adicionais sejam vividos com qualidade, é necessário que as pessoas tenham consciência da importância de cuidarem da sua saúde. Essa tarefa de promoção e de cuidado à saúde é de responsabilidade dos profissionais dessa área e do Estado (SOL et al., 2011).

Os idosos acumulam complicações decorrentes de doenças crônico-degenerativas múltiplas, que levam às incapacidades, à perda da autonomia e de qualidade de vida (QV), exigindo acompanhamento constante, com cuidados permanentes, medicação contínua e exames periódicos (CHAIMOWICZ, 1987; LIMA et al., 2009; REZENDE; GAEDE-CARRILLO; SEBASTIÃO, 2012).

O conceito de qualidade de vida relacionada à saúde (QVRS) assimila o bem-estar subjetivo, abrangendo ponderação cognitiva e ânimo, positivo e negativo (FENÁNDEZ-LÓPEZ; FERNÁNDEZ-FIDALGO; CIEZA, 2010), baseando-se em três princípios fundamentais: capacidade funcional, nível socioeconômico e satisfação pessoal. Além disso, a QVRS está 
vinculada ao componente como estado emocional, interação social, atividade intelectual, autoproteção de saúde, suporte familiar, valores culturais, éticos, religiosidade, estilo de vida, satisfação com o emprego e/ou com atividades diárias e o ambiente em que se vive (VECCHIA et al., 2005; GALISTEU et al., 2006). Nas últimas décadas, a avaliação da QVRS tem sido grandemente valorizada e usada no lugar de indicadores de saúde tradicionais de morbimortalidade, em função da necessidade de conhecer os resultados e a eficiência da aplicação de intervenções sobre o estado de saúde (LIMA; PORTELA, 2010).

Um dos instrumentos mais comumente usados para abordar o construto QVRS é a escala social Medical Outcomes Study 36-item Short-Form Health Survey (SF-36), elaborada pelo Medical Outcomes Study (MOS) e publicada, originalmente em inglês, em 1990. A escala é considerada um bom preditor de mortalidade e permite a análise de várias dimensões da saúde, a avaliação do impacto de doenças, assim como, os benefícios de tratamentos e terapias (LIMA et al., 2009).

A assistência à terceira idade é uma área de contato de muitas especialidades. Considerandose a diversidade e a complexidade dos indivíduos geriátricos, a atuação de uma equipe interdisciplinar torna-se fundamental, na medida em que participa, analisa e integra conhecimentos específicos de diversas áreas com o objetivo comum de promover e manter a saúde do idoso.

Neste sentido, foi criado em 2004, o Programa Terceira Idade: Vitalidade e Cidadania (PTI), com o intuito de promover uma maior integração entre indivíduos dessa faixa etária por meio de atividades físicas e sociais, na Universidade Federal de Ouro Preto (UFOP), localizada na cidade de Ouro Preto, Minas Gerais, Brasil (SOL et al., 2011).

Considerando o exposto, o presente trabalho teve como objetivo analisar a percepção dos idosos do PTI em relação à sua QVRS.

\section{Métodos}

Foi realizado um estudo de caráter transversal do tipo inquérito exploratório descritivo, com todos idosos $(\mathrm{n}=66)$ praticantes de atividades físicas no Centro Desportivo da UFOP residentes no município de Ouro Preto. Foram incluídos indivíduos com idade igual ou superior a 60 anos participantes do PTI, que aceitaram responder ao questionário após serem dadas as informações necessárias sobre a pesquisa. Depois de compreenderem os procedimentos da pesquisa, esses deram anuência por meio da assinatura do Termo de Consentimento Livre e Esclarecido.

Os dados primários coletados foram incluídos no banco de dados do PTI e sua confidencialidade foi garantida, sendo os idosos identificados por códigos.

Os dados foram coletados por meio de um questionário semiestruturado, que buscava identificar as características sociodemográficas e clínicas autorreferidas pelos participantes e o questionário SF-36. No Brasil, o instrumento SF-36 foi traduzido e adaptado às condições socioeconômicas e culturais de sua população, bem como, a demonstração de sua reprodutibilidade e validade por CICONELLI et al. (1999). Este questionário tem 11 questões, as quais reúnem 36 itens, sendo estruturado de forma que engloba 8 componentes (domínios ou dimensões), cuja pontuação de cada domínio varia de 0 a 100 pontos e reflete o pior e o melhor estado de saúde, respectivamente (LIMA et al., 2009).

Os resultados foram agrupados segundo a especificidade das respostas e os objetivos definidos e armazenados em planilha eletrônica do programa Excel, sendo analisados pelo programa estatístico STATA 9.0 e apresentados em frequência absoluta e relativa.

A pesquisa foi aprovada pelo Comitê de Ética em Pesquisa da UFOP sob o número Certificado de apresentação para apreciação científica (CAAE) 0016.238.000-09.

\section{Resultados}

As características sociodemográficas e clínicas dos idosos participantes do PTI estão descritas na Tabela 1 , tendo sido observado predomínio de mulheres $(\mathrm{n}=52 ; 78,8 \%)$, com faixa etária de 60 a 69 anos $(n=39 ; 59,1 \%)$, ensino fundamental como escolaridade referida $(n=46$; 
$69,7 \%)$, casados $(n=39 ; 59,0 \%)$. Quanto ao uso de medicamentos, 42,5\% $(n=28)$ deles relataram usar até dois.

Tabela 1 - Distribuição das variáveis sociodemográficas e número de medicamentos usados pelos idosos participantes

\begin{tabular}{|c|c|c|}
\hline Características & $\mathbf{n}$ & $\%$ \\
\hline \multicolumn{3}{|l|}{ Gênero } \\
\hline Masculino & 14 & 21,2 \\
\hline Feminino & 52 & 78,8 \\
\hline \multicolumn{3}{|l|}{ Idade (anos) } \\
\hline $60-64$ & 20 & 30,3 \\
\hline $65-69$ & 19 & 28,8 \\
\hline $70-74$ & 17 & 25,8 \\
\hline $75-79$ & 8 & 12,1 \\
\hline$\geq 80$ & 2 & 3,0 \\
\hline \multicolumn{3}{|c|}{ Escolaridade (anos estudados) } \\
\hline$\leq 8$ & 46 & 69,7 \\
\hline 9 a 11 & 17 & 25,8 \\
\hline$\geq 12$ & 3 & 4,5 \\
\hline \multicolumn{3}{|l|}{ Estado civil } \\
\hline Solteiro & 4 & 6,1 \\
\hline Divorciado & 4 & 6,1 \\
\hline Viúvo & 19 & 28,8 \\
\hline Casado & 39 & 59,0 \\
\hline \multicolumn{3}{|c|}{ Número de medicamentos usados } \\
\hline 0 & 11 & 16,6 \\
\hline 1 ou 2 & 17 & 25,8 \\
\hline 3 ou 4 & 21 & 31,8 \\
\hline$\geq 5$ & 17 & 25,8 \\
\hline
\end{tabular}

A Tabela 2 mostra a frequência dos problemas de saúde referidos pelos sujeitos. Note-se que estes problemas não são excludentes, ou seja, cada indivíduo poderia apresentar mais de uma morbidade, sendo a mais prevalente a hipertensão $(59,0 \%)$, e apenas $15,2 \%$ dos sujeitos referiram não apresentar nenhuma enfermidade.

Tabela 2 - Distribuição dos problemas de saúde relatados pelos idosos participantes do PTI em frequência absoluta e

\begin{tabular}{ccc}
\multicolumn{3}{c}{ relativa $(\mathrm{n}=66)$ em Ouro Preto } \\
\hline Problemas de saúde & $\mathbf{n}$ & $\mathbf{\%}$ \\
\hline Hipertensão & 39 & 59,0 \\
Dislipidemia & 15 & 22,7 \\
Diabetes & 9 & 13,6 \\
Alterações da tireoide & 8 & 12,1 \\
Outros* & 23 & 34,8 \\
Não relataram & 10 & 15,2
\end{tabular}

Obs: * Artrite $(n=6 ; 9,0 \%) ;$ Gastrite $(n=3 ; 4,5 \%) ;$ Neuropatia $(n=3 ; 4,5 \%)$; Osteoporose $(n=3 ; 4,5 \%)$; Glaucoma $(n=2 ; 3,0 \%)$; Labirintite $(n=2 ; 3,0 \%)$; Lúpus $(n=2 ; 3,0 \%)$; Catarata $(n=1 ; 1,5 \%)$; Hérnia de hiato $(n=1 ; 1,5 \%)$. Fonte: Autoria própria (2010).

A Tabela 3 apresenta os oito domínios avaliados pelo SF-36. Estes foram analisados, segundo mediana, $1^{\circ}$ e $3^{\circ}$ quartis para todos os domínios, exceto para dor e vitalidade. Esses dois apresentaram distribuição normal, deste modo, a análise foi realizada utilizando o valor das médias.

Observa-se que, excetuando-se o domínio dor, a pontuação mediana obtida foi acima de 70,0 , considerada boa para o grupo avaliado. O domínio aspectos sociais foi o que apresentou 
melhores resultados. Os domínios que demonstravam aspectos físicos, sociais e emocionais apresentaram pontuação de 100 para pelo menos 50,0\% dos sujeitos participantes da pesquisa.

Tabela 3 - Distribuição dos valores dos domínios avaliados pelo SF-36 apontados pelos idosos participantes do PTI

\begin{tabular}{|c|c|c|c|c|c|c|}
\hline Variável & $\begin{array}{l}\text { Méd } \\
\text { ia }\end{array}$ & $\begin{array}{r}\text { Desvio } \\
\text { Padrão } \\
\end{array}$ & $\begin{array}{l}\text { Quartil 1 } \\
(25 \%)\end{array}$ & $\begin{array}{l}\text { Mediana } \\
(\mathbf{5 0 \%})\end{array}$ & $\begin{array}{c}\text { Quartil 3 } \\
(75 \%)\end{array}$ & $\mathbf{p}$ \\
\hline Capacidade Funcional & 78,9 & 19,9 & 70,0 & 85,0 & 95,0 & 0,00001 \\
\hline Aspectos Físicos & 69,3 & 38,4 & 31,2 & 100,0 & 100,0 & 0,00380 \\
\hline Dor & 62,3 & 24,3 & 41,0 & 62,0 & 84,0 & 0,68830 \\
\hline Estado Geral de Saúde & 74,7 & 17,9 & 62,0 & 77,0 & 87,0 & 0,00700 \\
\hline Vitalidade & 73,1 & 17,3 & 60,0 & 75,0 & 88,8 & 0,42600 \\
\hline Aspectos Sociais & 89,2 & 18,6 & 87,5 & 100,0 & 100,0 & 0,00000 \\
\hline Aspectos Emocionais & 78,3 & 35,3 & 41,7 & 100,0 & 100,0 & 0,00010 \\
\hline Saúde Mental & 75,3 & 18,2 & 64,0 & 76,0 & 92,0 & 0,00330 \\
\hline
\end{tabular}

Fonte: Autoria própria (2010).

\section{Discussão}

A medida de QVRS possibilita o conhecimento de elementos concretos de avaliação clínica, ao considerar os aspectos da QV relacionados à presença de sintomas, incapacidades e limitações causados pelos problemas de saúde (FENÁNDEZ-LÓPEZ; FERNÁNDEZ-FIDALGO; CIEZA, 2010). Assim sendo, o SF-36 é um questionário apropriado para os objetivos dessa pesquisa.

A participação dos idosos nas atividades do PTI é intensa. As ausências, em geral, ocorrem por motivos alheios à vontade do frequentador, como ter de atender a alguma solicitação de um familiar ou por não estar se sentindo muito bem. O PTI apresenta alguns aspectos que favorecem a adesão e a permanência dos idosos no grupo: gratuito, fácil acesso ao local do programa, diversidade de atividades, horários compatíveis com a rotina diária, permite a ampliação das relações interpessoais, entre outros.

A maior participação de mulheres no PTI $(78,8 \%)$ é semelhante à frequência encontrada na literatura (GALISTEU et al., 2006; MOTA et al., 2006). Andreotti e Okuma (2003) citam como possível explicação o fato dos homens encontrarem, mais facilmente que as mulheres, oportunidades de se relacionarem socialmente fora do ambiente familiar como em clubes, jogos ou bares. O PTI apresenta um grupo de idosos 100,0\% ativo, predominando indivíduos de 60 a 69 anos $(59,1 \%)$. A faixa etária sexagenária apresenta melhores condições físicas para sair de casa e realizar atividades físicas: a literatura mostra que a proporção de indivíduos sedentários aumenta significativamente entre aqueles com idade mais avançada (NASCIMENTO et al., 2012).

Estudos apontam que era comum que mulheres jovens - hoje idosas - abandonassem os estudos formais quando da idade escolar, para auxiliarem nos afazeres domésticos familiares e se prepararem para o casamento (BIOSOLI-ALVES, 2000; RAMOS; VERAS; KALACHE, 1987).

A maioria dos sujeitos entrevistados nesse estudo era casada $(59,0 \%)$, taxa semelhante à encontrada em outras pesquisas (58,9\% por Lima et al. (2009); 60,2\% por Mota et al. (2006); 69,0\% por Pimenta et al. (2008)), mas bem diferente do estudo realizado por Toscano e Oliveira (2009), onde a predominância de viúvos (53,0\%) foi observada).

Em relação ao domínio do SF-36 referente à capacidade funcional, esta pode ser definida como o potencial apresentado pelos indivíduos para decidir e atuar em suas vidas de forma independente em seu cotidiano (RAMOS, 2003). Esta competência melhora significativamente quando o indivíduo realiza atividades físicas regularmente (VIDMAR et al., 2011).

Foi observado na Suécia que idosos com mais de 70 anos que caminhavam pelo menos 30 minutos todos os dias apresentaram uma capacidade física melhor do que aqueles que caminhavam menos de 30 minutos (CAETANO et al., 2008). Há uma correlação entre o aumento da idade com a diminuição da capacidade funcional, a qual está intimamente ligada à $\mathrm{QV}$, bem como, à capacidade de realização das atividades diárias (LOBO; SANTOS; CARVALHO, 2007). 
A relação entre capacidade funcional e aspectos físicos com a QVRS em idosos que apresentavam algum tipo de problema de saúde foi realizada na Turquia (OZTURK et al., 2011), sendo observado que o nível de capacidade funcional e QV eram influenciados pelo grau de mobilidade e pelo número de doenças crônicas nos idosos. Este estudo propôs que a realização de programas de reabilitação e exercícios físicos poderia levar a uma melhoria destes parâmetros, uma vez que aumentaria a disposição e diminuiria a percepção de dor, fatores que desempenham um papel importante na avaliação da QV.

Com o passar dos anos os idosos vão perdendo a capacidade de realizar as tarefas da vida diária e perdem sua capacidade física, cognitiva e funcional (NASCIMENTO et al., 2012). O estudo realizado por Nascimento e colaboradores (2012) mostrou que idosos com idade igual ou maior que 80 anos apresentaram uma capacidade funcional menor do que os que têm de 70 a 79 e, os da faixa etária de 60 a 69 anos tinham uma capacidade funcional bem maior do que os idosos com idade mais avançada. Isso pode explicar o fato da população estudada ser de idosos jovens.

Praticar exercício físico regularmente é um importante aliado na busca pela QV e bem-estar dos sujeitos (NASCIMENTO et al., 2012; VIDMAR et al., 2011), justificado pela melhora na composição corporal, redução de dores relatadas, aumento na densidade mineral óssea e na capacidade aeróbica, além de recuperar força, flexibilidade e resistência vascular (SANTOS; DANTAS; MOREIRA, 2011). No entanto, indivíduos que faziam atividades físicas mais rigorosas regularmente apresentaram escores mais elevados do que aqueles que praticavam atividades com gasto energético menor (TOSCANO; OLIVEIRA, 2009).

Nos dados encontrados no presente estudo observa-se que pelo menos 50,0\% dos indivíduos apresentou escore de 100 para o domínio aspectos físicos. O resultado já era previsível, uma vez que todos os participantes realizam atividade física regularmente (LIMA, 1999).

Em relação ao domínio dor, as queixas foram frequentes. $\mathrm{O}$ envelhecimento, na maioria das vezes, caracteriza-se pela elevada incidência de doenças incapacitantes, crônicas e degenerativas, bem como, de diferentes estados emocionais negativos, como a depressão, que podem comprometer a independência do indivíduo. Muitas dessas patologias, às vezes, são acompanhadas por dores, que podem interferir na sua QV, principalmente se forem nosologias de longa duração (DELLAROZA; PIMENTA; MATSUO, 2007).

Nesse estudo, o domínio referente à dor foi o que apresentou a menor média $(62,9)$. Este valor é inferior a 74,2 obtido na pesquisa de Lima e colaboradores (2009), realizada com idosos, de ambos os sexos, de quatro áreas do estado de São Paulo; e, superior a 54,8 encontrado em uma população de aposentados de Belo Horizonte (PIMENTA et al., 2008). No entanto, é intermediário para a média encontrada em investigação que avaliou os 8 domínios do SF-36 em indivíduos sedentários $(53,5)$ e ativos $(70,8)$ (MOTA et al., 2006) e indivíduos idosos menos e mais ativos 52,2 e 64,1, respectivamente (TOSCANO; OLIVEIRA, 2009). Desta forma, observa-se que a atividade física parece ter uma influência positiva no sentido de reduzir a dor nos estudos observados.

O resultado alerta para intervenções mais concretas em situações de dor que comprometam a autonomia e o bem-estar dos idosos. A dor crônica é fator limitante de funções físicas, funcionais e psíquicas, interferindo na relação social e na dinâmica familiar do indivíduo. Ela pode estar relacionada ao aumento da morbidade, onerando o Sistema de Saúde, estando associada a situações de sofrimento de longa duração, assim como, com o uso abusivo de medicamentos (ANDRADE; PEREIRA; SOUSA, 2006).

O fator idade tem considerável influência na QV. Como esperado, idosos possuem 'pior' estado de saúde que pessoas mais jovens. Uma pesquisa realizada no Brasil por Lima e colaboradores (2009) mostrou que não havia diferenças significativas entre os domínios dor e saúde mental, revelando que estas duas dimensões 'não são grandemente comprometidas pelo avanço da idade'.

O consumo elevado de medicamentos pelos idosos do presente estudo $(57,6 \%$ dos sujeitos utilizavam três ou mais medicamentos ao dia - Tabela 2) pode estar relacionado com o resultado do domínio dor. No entanto, o fato de ser um grupo que faz atividade física com regularidade, devidamente orientada, parece minimizar o impacto desta em sua QV (TOSCANO; OLIVEIRA, 
Em relação ao domínio estado geral de saúde, pelo menos 50,0\% dos idosos apresentaram a pontuação de 77,0 . Valores mais baixos $(59,0 \%)$ foram encontrados em um estudo que avaliou uma população de aposentados de Belo Horizonte, não participantes de nenhum grupo de convivência (PIMENTA et al., 2008). No entanto, comparando com os valores evidenciados nos outros domínios, o encontrado para estado geral de saúde foi menor do que os demais, com escore de 87,0 ou mais para somente $25,0 \%$ dos indivíduos. O estudo de Campolina, Dini e Ciconelli (2011) mostrou que a QVRS pode ser influenciada por aparecimento de morbidades pelos idosos, utilização de muitos medicamentos, ser do sexo feminino e presença de doenças crônicas, fatores que levam o individuo a ter uma autopercepção de QV ruim. Este estudo endossa os valores encontrados por essa pesquisa, uma vez que as características de ambos os estudos foram bastante semelhantes. Entende-se, então, que a magnitude do impacto e as dimensões mais afetadas variam de acordo com cada nosologia, mas é inegável que doenças crônicas influenciam de forma significativa na QVRS dos idosos.

Grande parte dos idosos participantes do PTI relatou apresentar doenças crônicas como hipertensão (59,0\%), dislipidemias $(22,7 \%)$ e diabetes $(13,6 \%)$ (Tabela 2). Esses problemas de saúde referidos parecem não influenciar de forma significativa na QV dos indivíduos entrevistados, uma vez que os escores encontrados nos demais domínios foram altos, como pode ser observado na Tabela 3. Este achado também foi notado no estudo de Muszalik e colaboradores (2011), na Polônia. Contudo, o estudo realizado em Fortaleza (avaliou os escores obtidos nos oito domínios do SF-36), com pacientes hipertensos cadastrados em uma Unidade Básica de Saúde da Família, mostrou que todos os escores apresentaram valores médios baixos (entre 53,6 e 69,4), comprometendo a percepção da QVRS (BRITO et al., 2008). Impacto maior ocorreu no estado geral de saúde e dor, com médias inferiores a 60,0 pontos para indivíduos de idades entre 25 a 65 anos, portanto, esperando-se que fossem obtidos melhores resultados.

O domínio vitalidade teve escore médio de 73,0, o qual está diretamente relacionado ao estado geral de saúde. É um valor superior à média encontrada no estudo de Mota e colaboradores (2006), com indivíduos idosos sedentários $(45,4)$ e ativos $(72,6)$; e, na pesquisa de Toscano e Oliveira (2009), com indivíduos idosos menos e mais ativos 56,9 e 72,9, respectivamente. Também superior a outros estudos com idosos que não participavam de grupos de convivência e não tinham prática regular de atividade física, cujas médias para este domínio foram 61,0 (LIMA et al., 2009) e 64,4 (PIMENTA et al., 2008), respectivamente.

Apesar de terem sido encontrados altos escores para os domínios vitalidade e estado geral de saúde, estes se apresentaram afetados provavelmente por doenças. Alerta-se para a necessidade de maiores considerações destes domínios nos programas de saúde para os idosos, visto que estes podem indicar cansaço, falta de energia e sentimentos negativos por parte dos participantes do PTI.

Como seres sociais, a saúde depende fundamentalmente do intercâmbio interpessoal em termos de recompensas materiais ou emocionais e de capacidade ou habilidade de aprendizado (FENÁNDEZ-LÓPEZ; FERNÁNDEZ-FIDALGO; CIEZA, 2010).

Contatos sociais entre idosos são fundamentais para boa QVRS (TOSCANO; OLIVEIRA, 2009). A avaliação da gravidade e a importância de um problema de saúde em idosas estão relacionadas mais à situação de enfrentá-lo do que pelo próprio problema, e este pode ser acentuado ou minimizado pela presença ou não de suporte familiar ou comunitário (GRUNDY; SLOGGETT, 2003).

A dinâmica psíquica do idoso é, ao mesmo tempo, rica e complicada. A afetividade, como capacidade de experimentar sentimentos e emoções e que dá a forma de expressá-los ao se relacionar com o mundo e consigo mesmo, pode influenciar nas dificuldades adaptativas do sujeito, tanto emocionais quanto fisiológicas, nas questões sociais, na dificuldade para aceitar o novo e na disposição geral para o relacionamento interpessoal ou com o mundo. Estes fatores são determinantes da QVRS em geriatria, no que diz respeito aos domínios emocionais e de saúde mental (FENÁNDEZ-LÓPEZ; FERNÁNDEZ-FIDALGO; CIEZA, 2010).

O equilíbrio emocional/mental ou psíquico do idoso depende, basicamente, de sua 
capacidade de adaptação à sua vivência presente em contraponto à vivência passada e das condições da realidade que o cercam. Este equilíbrio, dissertado por Lima (1999), citando o estudo publicado em 1965 por Cavan, e seu ajustamento ambiental dependem, principalmente, dos seguintes fatores: 'um contato social suficiente; uma ocupação cheia de significado; certa segurança social; um estado de saúde satisfatório', o que foi denominado de 'Teoria da Atividade'. Ou seja, a diminuição e o alento das relações sociais do idoso estão relacionados à mudança de estado, de ativo para inativo, a que os indivíduos estão sujeitos e à consequente redução dos rendimentos, devido à condição de aposentado (TORRES; MARQUES, 2008). Isso fornece subsídios para entender que quanto mais ativo o indivíduo se mostrar na velhice, maior será o seu grau de satisfação ou ajustamento social, o que está endossado pela afirmação de Andreotti e Okuma (2003): programas de atividades aeróbicas se associam com respostas psicossociais mais elevadas e com baixos níveis de sintomas depressivos em indivíduos que praticam regularmente atividades físicas.

Medidas de intervenção visando identificar causas tratáveis de déficit cognitivo e de perda de independência no dia-a-dia deveriam tornar-se prioridade do sistema de saúde, dentro de uma perspectiva de reestruturação programática realmente sintonizada com a saúde e o bem-estar da crescente população de idosos. O objetivo principal do sistema deve ser a manutenção da capacidade funcional do idoso, mantendo-o ativo na comunidade, pelo maior tempo possível, gozando ao máximo sua independência (RAMOS, 2003; LOBO; SANTOS; CARVALHO, 2007).

$\mathrm{O}$ fato dos domínios aspectos emocionais e mentais não terem escores tão baixos e apresentarem valores aproximados, pode ser explicado pela adaptação às condições impostas pelas doenças que levaram os idosos a investirem em ações para melhorar a sua QV e estes tiveram o acompanhamento do PTI para essa promoção à saúde.

\section{Considerações finais}

Os resultados do presente estudo permitiram conjecturar que grande parcela dos idosos avaliados era bastante ativa e conhecia os benefícios da prática regular de exercícios físicos. Embora os idosos do PTI apresentassem problemas de saúde crônicos e fizessem uso de polifarmácia, os escores de QVRS foram bons, talvez devido à regularidade das atividades físicas dentro do conjunto de ações e estímulo da assistência multidisciplinar ofertada.

Ainda considerando os idosos pertencentes ao PTI, a busca por novos modelos de envelhecimento saudável pretende, fundamentalmente, fugir dos aspectos engessados ao longo da história, que definem a figura do 'velho' como indivíduo inútil, improdutivo e, até, de presença indesejável. Essa relação, porém, se define sobre novos moldes na atualidade, de modo que o primeiro e principal obstáculo para se assumir um modelo de velhice mais gratificante se impõe internamente, através da superação individual de concepções antiquadas do que é ser idoso.

\section{Agradecimentos}

Núcleo de Estudos sobre Envelhecimento (NesE) e Pró-Reitoria de Extensão da Universidade Federal de Ouro Preto (ProEx).

\section{Referências}

ANDRADE, F. A.; PEREIRA, L. V.; SOUSA, F. A. E. F. Mensuração da dor no idoso: uma revisão. Revista Latino-Americana de Enfermagem, v. 14, n. 2, p. 271-276, mar./abr. 2006. Disponível em: 〈http://www.scielo.br/pdf/rlae/v14n2/v14n2a18.pdf > . Acesso em: 25 ago. 2014.

ANDREOTTI, M. C.; OKUMA, S. S. Perfil sócio-demográfico e de adesão inicial de idosos ingressantes em um programa de educação física. Revista Paulista de Educação Física, v. 17, n. 2, p. 142-153. jul./dez. 2003. Disponível em: <http://citrus.uspnet.usp.br/eef/uploads/arquivo/v17\%20n2\%20artigo6.pdf >. Acesso em: 01 set. 2014. 
BIOSOLI-ALVES, Z. M. M. Continuidades e rupturas no papel da mulher brasileira no século XX. Psicologia: Teoria e Pesquisa, v. 16, n. 3, p. 233-239, set./dez. 2000. Disponível em: <http://www.scielo.br/pdf/ptp/v16n3/4810.pdf>. Acesso em: 25 ago. 2014.

BRITO, D. M. S.; ARAÚJO, T. L.; GALVÃO, M. T. G.; MOREIRA, T. M. M.; LOPES, M. V. O. Quality of life and perception of illness among individuals with high blood pressure. Cadernos de Saúde Pública, v. 24, n. 4, p. 933-940, abr. 2008. Disponível em:

<http://www.scielosp.org/scielo.php?pid=S0102-311X2008000400025\&script=sci_arttext $>$. Acesso em: 25 ago. 2014.

CAETANO, J. A.; COSTA, A. C.; SANTOS, Z. M. S. A.; SOARES, E. Descrição dos fatores de risco para alterações cardiovasculares em um grupo de idosos. Texto \& Contexto Enfermagem, v. 17, n. 2, p. 327-335, 2008. Disponível em: 〈http://www.scielo.br/pdf/tce/v17n2/15.pdf >. Acesso em: 01 set. 2014.

CAMPOLINA, A. G.; DINI, P. S.; CICONELLI, R. M. Impacto da doença crônica na qualidade de vida de idosos da comunidade em São Paulo (SP, Brasil). Ciência \& Saúde Coletiva, v. 16, n. 6, p. 2919-2925, 2011. Disponível em: 〈http://www.scielo.br/pdf/csc/v16n6/29.pdf〉. Acesso em: 01 set. 2014.

CHAIMOWICZ, F. A saúde dos idosos brasileiros às vésperas do século XXI: problemas, projeções e alternativas. Revista de Saúde Pública, v. 31, n. 2, p. 184-200, 1987. Disponível em: <http://www.scielo.br/scielo.php?pid=S0034-89101997000200014\&script=sci_arttext $>$. Acesso em: 25 ago. 2014.

CICONELLI, R. M.; FERRAZ, M. B.; SANTOS, W.; MEINÃO, I.; QUARESMA, M. R. Tradução para a língua portuguesa e validação do questionário genérico de avaliação de qualidade de vida SF36 (Brasil SF-36). Revista Brasileira de Reumatologia, v. 39, p. 143-150, 1999. Disponível em: <http://lava.med.br/MESTRADO/VASCULAR/2005/Artigos_Revista/Modulo_XXVI/390301.pdf> . Acesso em: 01 set. 2014.

DELlAROZA, M. S. G.; PIMENTA, C. A. M.; MATSUO, T. Prevalência e caracterização da dor crônica em idosos não institucionalizados. Cadernos de Saúde Pública, v. 23, n. 5, p. 1151-1160, 2007. Disponível em: <http://www.scielo.br/scielo.php?pid=S0102-

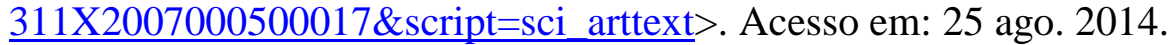

FERNÁNDEZ-LÓPEZ, J. A.; FERNÁNDEZ-FIDALGO, M.; CIEZA, A. Los conceptos de salud, calidad de vida y funcionamiento desde El punto de vista de la Clasificación Internacional de Funcionamiento. Revista Española de Salud Pública, v. 84, n. 2, p. 169-184, mar./abr. 2010. Disponível em: $<$ http://scielo.isciii.es/scielo.php?pid=S113557272010000200005\&script=sci_arttext $>$. Acesso em: 10 set. 2014.

GALISTEU, K. J.; FACUNDIM, S. D.; RIBEIRO, R. C. H. M.; SOLER, Z. A. S. G. Qualidade de vida de idosos de um grupo de convivência com a mensuração da escala de Flanagan. Arquivos de Ciências da Saúde, v. 13, n. 4, p. 209-214, out./dez. 2006. Disponível em: $<$ http://www.cienciasdasaude.famerp.br/racs_ol/vol-13-4/Famerp\%2013(4)\%20ID\%20187\%20\%2016.pdf $>$. Acesso em: 10 set. 2014.

GRUNDY, E.; SLOGGETT, A. Health inequilities in the older population: the role of personal capital, social resources and socio-economic circumstances. Social Science \& Medicine, v. 56, p. 935-947, 2003. Disponível em:

<http://www.sciencedirect.com/science/article/pii/S027795360200093X >. Acesso em: 01 set. 2014. 
LIMA, M. A. A Gestão da experiência de envelhecer em um programa para a terceira idade: a UnATI/UERJ. Textos sobre Envelhecimento - UnATI/UERJ, Rio de Janeiro, ano 2, n. 2, p. 23 63, 2. sem. 1999. Disponível em: 〈http://bases.bireme.br/cgi-

bin/wxislind.exe/iah/online/?IsisScript=iah/iah.xis\&src=google\&base=LILACS\&lang=p\&nextActi on=lnk\&exprSearch=291183\&indexSearch=ID> . Acesso em: 25 ago. 2014.

LIMA, M. G.; BARROS, M. B. A. B.; CÉSAR, C. L. G. C.; GOLDBAUM, M.; CARANDINA, L.; CICONELLI, R. M. Health related quality of life among the elderly: a population-based study using SF-36 survey. Cadernos de Saúde Pública, v. 25, n. 10, p. 2159-2167, out. 2009. Disponível em: <http://www.scielo.br/scielo.php?script=sci_arttext\&pid=S0102-311X2009001000007>. Acesso em: 10 set. 2014.

LIMA, M. J. B.; PORTELA, M. C. Elaboração e avaliação da confiabilidade de um instrumento para medição da qualidade de vida relacionada à saúde de idosos independentes. Cadernos de Saúde Pública, v. 26, n. 8, p.1651-1662, ago. 2010. Disponível em:

<http://www.scielo.br/scielo.php?pid=S0102-311X2010000800018\&script=sci_arttext $>$. Acesso em: 10 set. 2014.

LOBO, A.; SANTOS, M. P.; CARVALHO, J. Anciano institucionalizado: calidad de vida y funcionalidad. Revista Española de Geriatría y Gerontología, v. 42, n. 1, p. 22-26, 2007. Disponível em: <http://zl.elsevier.es/es/revista/revista-espanola-geriatria-gerontologia-124/ancianoinstitucionalizado-calidad-vida-funcionalidad-13110113-originales-2007>. Acesso em: 01 set. 2014.

MOTA, J.; RIBEIRO, J. L.; CARVALHO, J.; MATOS, M. G. Atividade física e qualidade de vida associada à saúde em idosos participantes e não participantes em programas regulares de atividade física. Revista Brasileira de Educação Física e Esporte, v. 20, n. 3, p. 219-225, jul./set. 2006. Disponível em: 〈http://www.revistas.usp.br/rbefe/article/viewFile/16629/18342〉. Acesso em: 01 set. 2014.

MUSZALIK, M.; DIJKSTRA, A.; KEDZIORA-KORNATOWSKA, K.; ZIELINSKAWIECZKOWSKA, H.; KORNATOWSKI, T. Independence of elderly patients with arterial hypertension in fulfilling their needs, in the aspect of functional assessment and quality of life (QoL). Archives of Gerontology and Geriatrics, v. 52, p. 204-209, 2011. Disponível em: <http://www.aggjournal.com/article/S0167-4943(10)00289-X/abstract>. Acesso em: 10 set. 2014.

NASCIMENTO, C. M.; RIBEIRO, A. Q.; COTTA, R. M. M.; ACURCIO, F. A.; PEIXOTO, S. V.; PRIORE, S. E.; FRANCESCHINI, S. C. C. Factors associated with functional ability in Brazilian elderly. Archives of Gerontology and Geriatrics, n. 54, p. 89-94, 2012. Disponível em: <http://www.ncbi.nlm.nih.gov/pubmed/21925745>. Acesso em: 10 set. 2014.

OZTURK, A.; SIMSEK, T. T.; YUMIN, E. T.; SERTEL, M.; YUMIN M. The relationship between physical, functional capacity and quality of life (QoL) among elderly people with a chronic disease. Archives of Gerontology and Geriatrics, v. 53, p. 278-283, 2011. Disponível em: <http://www.aggjournal.com/article/S0167-4943(10)00335-3/abstract>. Acesso em: 01 set. 2014.

PIMENTA, F. A. P.; SIMIL, F. F.; TORRES, H. O. G.; AMARAL, C. F. S.; REZENDE, C. F.; COELHO, T. O.; REZENDE, N. A. Avaliação da qualidade de vida de aposentados com a utilização do questionário SF-36. Revista da Associação Médica Brasileira, v. 54, n. 1, p. 55-60, 2008. Disponível em: <http://www.scielo.br/pdf/ramb/v54n1/21.pdf >. Acesso em: 25 ago. 2014. 
RAMOS, L. R. Fatores determinantes do envelhecimento saudável em idosos residentes em centro urbano: Projeto Epidoso São Paulo. Cadernos de Saúde Pública, v. 19, n. 3, p. 793-798, maio/jun. 2003. Disponível em: <http://www.scielo.br/scielo.php?script=sci_arttext\&pid=S0102311X2003000300011>. Acesso em: 25 ago. 2014.

RAMOS, L. R.; VERAS, R. P.; KALACHE, A. Envelhecimento populacional: uma realidade brasileira. Revista de Saúde Pública, v. 21, n. 3, p. 211-224, 1987. Disponível em: <http://www.saude.sp.gov.br/resources/ses/perfil/profissional-da-saude/grupo-tecnico-de-acoesestrategicas-gtae/saude-da-pessoa-idosa/artigos-eteses/envelhecimento_populacional_uma_realidade_brasileira.pdf > . Acesso em: 25 ago. 2014.

REZENDE, C. P.; GAEDE-CARRILLO, M. R. G.; SEBASTIÃO, E. C. O. Queda entre idosos no Brasil e sua relação com o uso de medicamentos: revisão sistemática. Cadernos de Saúde Pública, v. 28, n. 12, p. 2223-2235, dez. 2012. Disponível em:

$<$ http://www.scielo.br/scielo.php?script=sci_arttext\&pid=S0102-311X2012001400002 > Acesso em: 25ago. 2014.

SANTOS, C. A. S.; DANTAS, E. E. M.; MOREIRA, M. H. R. Correlation of physical aptitude; functional capacity, corporal balance and quality of life (QoL) among elderly women submitted to a post-menopausal physical activities program. Archives of Gerontology and Geriatrics, n. 53, p. 344-349, 2011. Disponível em: <http://www.aggjournal.com/article/S0167-4943(10)003432/abstract>. Acesso em: 10 set. 2014.

SOL, N. A. A.; PRADO, I. B. H.; GAEDE-CARRILLO, M. R. G.; SERRA, C. O. P.; SEBASTIÃO, E. C. O.; SANTOS, M. A.; NIMER, M.; FIGUEIREDO, R. C. Programa terceira idade: vitalidade e cidadania - uma proposta de atenção interdisciplinar. Saúde Coletiva, v. 8, n. 54, p. 239-243, 2011. Disponível em: 〈http://www.redalyc.org/pdf/842/84221108004.pdf〉. Acesso em: 25 ago. 2014.

TORRES, M.; MARQUES, E. Envelhecimento activo: um olhar multidimensional sobre a promoção da saúde. Estudo de caso em Viana do Castelo. In: CONGRESSO PORTUGUÊS DE SOCIOLOGIA. Mundos Sociais: saberes e práticas, 6., 2008, Lisboa. Anais... Lisboa: CONGRESSO PORTUGUÊS DE SOCIOLOGIA, 2008.

TOSCANO, J. J. O.; OLIVEIRA, A. C. C. Qualidade de vida em idosos com distintos níveis de atividade física. Revista Brasileira de Medicina do Esporte, v. 15, n. 3, p. 169-173, maio/jun. 2009. Disponível em: 〈http://www.scielo.br/pdf/rbme/v15n3/a01v15n3.pdf >. Acesso em: 01 set. 2014.

VECCHIA, R. D.; RUIZ, T.; BOCCHI, S. C. M.; CORRENTE, J. E. Qualidade de vida na terceira idade: um conceito subjetivo. Revista Brasileira de Epidemiologia, v. 8, n. 3, p. 246-252, 2005. Disponível em: $<$ http://www.scielo.br/scielo.php?script=sci_arttext\&pid=S1415790X2005000300006>. Acesso em: 10 set. 2014.

VIDMAR, M. F.; POTULSKI, A. P.; SACHETTI, A.; SILVEIRA, M. M.; WIBELINGER, L. M. Atividade física e qualidade de vida em idosos. Revista Saúde e Pesquisa, v. 4, n. 3, p. 417-424, set./dez. 2011. 